\title{
Tumor Necrosis Factor Receptor Expression and Signaling in Renal Cell Carcinoma
}

\author{
Rafia S. Al-Lamki, ${ }^{*}$ Timothy J. Sadler, ${ }^{*}$ \\ Jun Wang, ${ }^{*}$ Martin J. Reid, ${ }^{*}$ Anne Y. Warren, ${ }^{\dagger}$ \\ Mehregan Movassagh, ${ }^{\ddagger}$ Wanhua Lu, ${ }^{*}$

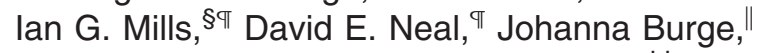 \\ Peter Vandenebeele, ${ }^{* \star}$ Jordan S. Pober ${ }^{\dagger \dagger}$ \\ and John R. Bradley*

\begin{abstract}
From the Departments of Medicine, ${ }^{*}$ and Pathology, ${ }^{\dagger}$ and Division of Cardiovascular Medicine, ${ }^{*}$ University of Cambridge, for Molecular Medicine Norway, ${ }^{\S}$ Nordic European Molecular Biology Laboratory (EMBL), Oslo, Norway; the Uro-Oncology Research Group, "T the Cancer Research UK Cambridge Research Institute, Li Ka Shing Centre, Cambridge, United Kingdom; the Department of Urology," Addenbrooke's Hospital, Cambridge, United Kingdom; the Department of Molecular Biology, ** State University of Ghent, Ghent, Belgium; and the Departments of Immunobiology, Pathology, and Dermatology, ${ }^{\text {t+ }}$ Yale University School of Medicine, New Haven, Connecticut
\end{abstract} \\ Addenbrooke's Hospital, Cambridge, United Kingdom; the Centre
}

Clear cell renal cell carcinoma (ccRCC), a tubular epithelial cell (TEC) malignancy, frequently secretes tumor necrosis factor (TNF). TNF signals via two distinct receptors (TNFRs). TNFR1, expressed in normal kidney primarily on endothelial cells, activates apoptotic signaling kinase 1 and nuclear factor- $\kappa \mathrm{B}$ (NF$\kappa B)$ and induces cell death, whereas TNFR2, inducibly expressed on endothelial cells and on TECs by injury, activates endothelial/epithelial tyrosine kinase (Etk), which trans-activates vascular endothelial growth factor receptor 2 (VEGFR2) to promote cell proliferation. We investigated TNFR expression in clinical samples and function in short-term organ cultures of ccRCC tissue treated with wild-type TNF or specific muteins selective for TNFR1 (R1-TNF) or TNFR2 (R2-TNF). There is a significant increase in TNFR2 but not TNFR1 expression on malignant TECs that correlates with increasing malignant grade. In ccRCC organ cultures, R1-TNF increases TNFR1, activates apoptotic signaling kinase and $\mathrm{NF}-\kappa \mathrm{B}$, and promotes apoptosis in malignant TECs. R2-TNF increases TNFR2, activates NF- $\boldsymbol{k}$, Etk, and VEGFR2 and increases entry into the cell cycle. Wild-type TNF induces both sets of responses. R2-TNF actions are blocked by pretreatment with a
VEGFR2 kinase inhibitor. We conclude that TNF, acting through TNFR2, is an autocrine growth factor for ccRCC acting via Etk-VEGFR2 cross-talk, insights that may provide a more effective therapeutic approach to this disease. (Am J Pathol 2010, 177:943-954; DOI: 10.2353/ajpath.2010.091218)

Despite increased understanding of the pathological mechanisms involved, renal cell carcinoma (RCC) tends to be resistant to conventional treatment with radioand/or chemotherapy. Clear cell RCC (ccRCC), which is the most common form, is associated with inactivating mutations of the von Hippel-Lindau (VHL) tumor suppressor gene. Functional loss of the $\mathrm{VHL}$ protein ( $\mathrm{pVHL}$ ) is associated with increased transcription of hypoxia-inducible genes, such as vascular endothelial growth factor (VEGF), and inhibition of VEGF has proven to be of clinical benefit in ccRCC. ${ }^{1} \mathrm{pVHL}$ also represses translation of tumor necrosis factor (TNF) mRNA. TNF expression is increased in $\mathrm{pVHL}$-deficient cells, ${ }^{2}$ and phase II clinical trials using infliximab (anti-TNF monoclonal antibody) have shown promising results in patients with advanced RCC. ${ }^{3}$

TNF signals through two distinct cell surface receptors, designated TNFR1 and TNFR2, and the ability of each TNFR to interact with both identical and unrelated downstream signaling molecules explains their shared and diverse functions. TNFR1 activates apoptosis signal-regulating kinase 1 (ASK1), a mitogen-activated protein kinase kinase kinase that mediates apoptosis and activates downstream signaling molecules such as c-Jun $\mathrm{N}$-terminal kinase and p38 mitogen-activated protein kinase. TNFR2 activates endothelial/epithelial tyrosine ki-

Supported by Addenbrooke's Charitable Trust (grant KDD/9730 to R.S.A.), Kidney Research (grant UK RP31/2/06 to J.R.B.), National Institute for Health Research Cambridge Biomedical Research Centre Award (J.R.B.), and National Institutes of Health (grant R01-HL36003 to J.S.P.).

Accepted for publication April 23, 2010.

Supplemental material for this article can be found on http://ajp. amjpathol.org.

Address reprint requests to Dr. Rafia S. Al-Lamki, Department of Medicine, University of Cambridge, Level 5, Addenbrooke's Hospital, Cambridge CB2 OQQ, UK. E-mail: rsma2@hermes.cam.ac.uk. 
nase (Etk), a member of the Btk nonreceptor tyrosine kinase family implicated in cell adhesion, migration, proliferation, and survival. In endothelial cells, Etk signaling involves tyrosine phosphorylation of the intracellular regions of VEGFR2, resulting in a VEGF-independent proangiogenic response mediated through a signaling cascade including phosphatidylinositol 3-kinase and Akt (also known as protein kinase B). ${ }^{4}$

Expression of TNFRs is highly regulated in human kidney, ${ }^{5}$ and we hypothesized that regulated TNFR expression may be an important determinant of growth and progression of ccRCC. To investigate this we have i) assessed TNFR expression on normal and malignant (m) TECs in different grades of ccRCC (which represent 75\% of all RCC cases) $)^{6}$ using immunofluorescence microscopy and a panel of established immunomarkers for mTECs and ii) examined the effects of wild-type (wt) TNF and TNFR-specific muteins (R1-TNF and R2-TNF) both on the activation of associated signaling molecules and on cellular responses such as cell cycle entry and death in cCRCC tissue using short-term organ cultures. Here we report that TNFR2 is induced and up-regulated in mTECs and its increased expression correlates with malignant grade. We also show that in short-term organ cultures of cCRCC, R1-TNF and wtTNF increase TNFR1, activate ASK1 and nuclear factor $-\kappa \mathrm{B}(\mathrm{NF}-\kappa \mathrm{B})$, and promote apoptosis, whereas R2-TNF and wtTNF increase TNFR2, activate phospho-Etk ${ }^{\mathrm{PT} y \mathrm{r} 4 \mathrm{O}}, \mathrm{NF}-\kappa \mathrm{B}$, and phospho-VEGFR2 ${ }^{\text {PY1054-1059, }}$, and promote cell cycle entry in mTECs. VEGF also induces phosphoVEGFR2 ${ }^{\text {pY1054-1059 }}$ but not activation of phospho-Etk ${ }^{\text {PTyr4O }}$, suggesting that the signals induced by TNFR2 and VEGFR2 are distinct because only the former leads to activation of Etk. However, a VEGFR2-selective kinase inhibitor blocks cellular responses to both R2-TNF and VEGF. These observations suggest that TNF, acting selectively through a TNFR2/Etk/VEGFR2 pathway, is an autocrine growth factor that may contribute to cCRCC progression.

\section{Materials and Methods}

\section{Tissue Collection}

Experiments using human tissue were performed with the written informed consent of patients and approval of the local ethics committee and Addenbrooke's Hospital Tissue Bank. RCC tissue was obtained immediately after surgical excision and classified according to histological cell type (clear cell, papillary, chromophobe cell, sarcomatoid, or collecting duct). ${ }^{7}$ For this study, we have used cCRCC, graded according to the four-tiered nuclear grading system ${ }^{8}$ and pathologically staged based on the TNM classification. ${ }^{9}$ Tissue samples from 76 patients were scored as Fuhrman grade 1 ( $n=19$; pT1 $=5 \%$, pT1a $=26 \%$, pT1b $=5 \%$, pT2 $=32 \%$, pT2a $=16 \%$; male $=53 \%$, female $=47 \%$; mean age \pm SD of $60.4 \pm$ 9.5 years; tumor size $=6.3 \pm 1.7 \mathrm{~cm}$ ), Fuhrman grade 2 $(n=19 ; \mathrm{pT} 1=26 \%, \mathrm{pT} 1 \mathrm{a}=11 \%, \mathrm{pT} 1 \mathrm{~b}=11 \%, \mathrm{pT} 2=$ $26 \%$, pT3b $=26 \%$; male $=58 \%$, female $=42 \%$; mean age \pm SD of $60.1 \pm 9.0$ years; tumor size $=6.1 \pm 9.0$ cm), Fuhrman grade 3 ( $n=19 ;$ pT1 $=16 \%$, pT1a $=5 \%$, pT2 $=21 \%$, pT2a $=11 \% ;$ pT3a $=21 \%$, pT3b $=16 \%$, pT4 $=10 \%$; male $=68 \%$; female $=52 \%$; mean age \pm SD of $55.6 \pm 13.0$ years; tumor size $=7.5 \pm 1.8 \mathrm{~cm})$, and Fuhrman grade 4 ( $n=19$; pT1 $=5 \%$, pT1a $=16 \%$, pT1b $=11 \%$, pT2 $=5 \%$, pT2a $=11 \%$, pT3a $=21 \%$, pT4 $=31 \%$; male $=72 \%$, female $=26 \%$; mean age \pm SD of $67.0 \pm 8.0$ years; tumor size $=7.4 \pm 1.0 \mathrm{~cm}$ ). Samples from histopathologically normal kidney cortex remote from the tumor site were obtained from 50 tumor-bearing kidneys. Samples were encapsulated in Cryo-M-Bed embedding compound (Bright Instrument Co. Ltd., Huntingdon, Cambridgeshire, UK) and frozen in liquid nitrogen or immersed in 4\% formaldehyde (BDH Merck Ltd., Lutterworth, Leics, UK) in 0.1 M PIPES buffer, $\mathrm{pH} 7.6$, for 1.5 hours at $4^{\circ} \mathrm{C}$ and paraffin wax-embedded for immunohistochemical studies. Paraffin sections from each batch of tissue were stained with $\mathrm{H} \& \mathrm{E}$, and the diagnosis in all cases was verified independently by two experienced pathologists and was based entirely on examination of routinely stained slides.

\section{ccRCC Organ Cultures}

Additional pieces of renal tissue from six nontumoral kidneys and from six Fuhrman grade 1 ccRCCs were obtained immediately from surgically excised specimens. Duplicate $1-\mathrm{mm}^{3}$ fragments of tissue were placed in a Corning flat-bottomed 96-well tissue culture plate (Appleton Woods Limited, Birmingham, UK) and immediately immersed in Medium 199 containing 10\% heatinactivated fetal calf serum (TCS, Botolph Claydon, Bucks, UK) and $2.2 \mathrm{mmol} / \mathrm{L}$ glutamine as reported previously. ${ }^{10,11}$ Multiple randomized samples from each patient were used to obtain parallel group comparisons and to assess the reliability and reproducibility of these assays. Tissues were incubated in media alone (untreated) or pretreated with a VEGFR2-selective tyrosine kinase inhibitor (SU1498, catalog 572888, Merck Chemicals Ltd., Nottingham, UK) at a concentration of 25, 50, or 100 $\mu \mathrm{g} / \mathrm{ml}$ for 30 minutes at $37^{\circ} \mathrm{C}$ followed by stimulation with either wild-type recombinant human TNF (AMS Biotechnology, Abingdon Oxon, UK) or recombinant human VEGF (R\&D Systems, Oxford, UK) or a combination of TNF and VEGF (all used at $10 \mathrm{ng} / \mathrm{ml}$ ) or $1 \mu \mathrm{g} / \mathrm{ml}$ of recombinant mutations of the wtTNF sequence, which will enable the mutated protein ("mutein") to bind selectively to either of the TNFR subtypes. ${ }^{12}$ The specific double mutation of R32W, S86T (here termed R1-TNF) allows selective activation of TNFR1, whereas the D143N, A145R (termed R2-TNF) double mutation allows selective activation of the TNFR2 subtype only. In addition, some organ cultures were in parallel incubated either with wtTNF or TNF-selective muteins at various time points (5 minutes, 30 minutes, 1 minute, 2 hours, and 4 hours) or with various dose of wtTNF $(0.1,1,3$, and $10 \mathrm{ng} / \mathrm{ml})$ for 3 hours. Cultures were harvested and either snap-frozen in liquid nitrogen or fixed in $4 \%$ paraformaldehyde for paraffin wax-embedding. 


\section{General Reagents and Antibodies}

Antibodies used were goat anti-TNFR1, mouse anti-TNFR1, and mouse anti-TNFR2 (all from R\&D Systems, Abington, UK), rabbit anti-TNFR2, rabbit anti-Etk/Bmx, and mouse anti-VEGF (all from Autogen Bioclear, Wiltshire, UK), mouse anti-RCC marker (RCC-Ma) and mouse anti-CD10 (both from Novocastra, Newcastle-on-Tyne, UK), mouse anti-pancytokeratin (pan-CK) and mouse anti-vimentin (both from DakoCytomation, Ely, UK), rabbit anti-ASK1-pSer967 or -pThr845 and anti-phospho-Etk ${ }^{\text {pTyr4O }}$, rabbit anti-cleaved active caspase- $3^{\mathrm{p} 175}$, and rabbit anti-NF- $\mathrm{B}^{\mathrm{P}-\mathrm{S} 276-\mathrm{p} 65}$ (all from Cell Signaling, Hertfordshire, UK), rabbit anti-phospho-VEGFR2 ${ }^{\text {pY1054-59, }}$, rabbit anti-histone-H3 $3^{\text {phospho-S10, }}$ and rabbit anti-ki67 (all from Abcam, Cambridge, UK), and anti-intercellular adhesion molecule (ICAM)-1 (Millipore, Watford, UK). Secondary antibodies included Northern light $(\mathrm{NL})^{498}$-conjugated anti-goat or anti-rabbit and $\mathrm{NL}^{557}$-conjugated anti-mouse or anti-rabbit (R\&D Systems). Tyramide Signal Amplification was performed with fluorescein-tyramide, blocking reagent, and amplification diluents (DuPont NEN, Boston, MA). Primary antibodies were used at 1:50 and secondary antibodies at 1:100 dilution. Nuclei were detected using To-PRO-3' iodide (Molecular Probes, Eugene, OR). Antigen retrieval for RCC-Ma was done by chymotrypsin ( $\mathrm{pH} \mathrm{7.8)}$ at $37^{\circ} \mathrm{C}$ (Sigma-Aldrich, Gillingham, Dorset, UK); antigen retrieval for pan-CK, CD10, pH3 ${ }^{\text {phospho-S10, active-caspase- }}$ $3^{\mathrm{Asp} 175}$, and $\mathrm{NF}-\kappa^{\mathrm{P}-\mathrm{S} 276-\mathrm{p} 65}$ was done by pressure cooker with $0.01 \mathrm{~mol} / \mathrm{L}$ citric acid buffer $(\mathrm{pH}$ 6.0) for 2 minutes. Frozen sections were permeabilized in methanol at $-20^{\circ} \mathrm{C}$ for 5 minutes before immunostaining.

\section{Immunohistochemistry}

\section{Detection of TNFR Protein and RCC-Ma or CD10 or Pan-CK or Vimentin in ccRCC Tissue}

Paraffin wax sections of nontumoral kidney and ccRCC were coimmunostained using our previously described protocol. ${ }^{11}$ In brief, dewaxed sections were immunostained for TNFR1 or TNFR2 and RCC-Ma or CD10 or pan-CK or vimentin (this panel of immunomarkers allow clear cell carcinomas of the kidney to be distinguished with a high degree of certainty and is a useful adjunct to histological examination). ${ }^{13-15}$ For a negative control, the primary antibody was omitted.

\section{Detection of TNFR Protein and Associated Signaling Molecules in ccRCC Organ Culture}

Dewaxed sections of nontumoral kidney and cCRCC organ cultures (untreated and wtTNF-, R1-TNF-, or R2-TNFtreated) were immunostained for TNFR1, TNFR2, VEGF, and anti-ASK1-pSer967 or -pThr845, or anti-phospho-Etk ${ }^{\text {P }}$ Tyr40, or anti-phospho-VEGFR2 ${ }^{\text {pY1054-59. Parallel sections }}$ were labeled for cleaved active caspase- $3^{\mathrm{p} 175}$, anti-histone$\mathrm{H} 3^{\text {phospho-S10 }}, \mathrm{Ki}-67$, and NF- $\mathrm{B}^{\mathrm{P}-\mathrm{S} 276-\mathrm{p} 65}$ followed by ToPRO-3' iodide as described previously. ${ }^{11}$ Some cultures that stained for $\mathrm{NF}-\kappa \mathrm{B}^{\mathrm{P}-\mathrm{S} 276-\mathrm{p} 65}$ were further stained with ICAM-1 (CD-54) as consensus sequences for the NF- $\kappa \mathrm{B}$ have been described in the ICAM-1 $5^{\prime}$ flanking region. ${ }^{16}$ In addition, cultures pretreated with or without VEGFR2-specific tyrosine kinase inhibitor were immunostained for anti-phospho-Etk ${ }^{\text {pTyr4O }}$ or anti-phospho-VEGFR2 ${ }^{\text {PY1054-59 }}$ or anti-histone-H3 $3^{\text {phospho-S10, }}$ and some sections were costained for anti-phospho-Etk ${ }^{\text {Tyr40 }}$ or anti-phosphoVEGFR2 ${ }^{\text {PY1054-59 }}$ using a Tyramide Amplification technique. ${ }^{17}$ For a negative control, the primary antibody was omitted. Sections were mounted in VECTASHIELD Mounting Media (Vector Laboratories Ltd., Peterborough, UK) and examined using a Leica TCSPE confocal laser scanning microscope (Leica Microsystems, Milton Keynes, UK).

\section{Immunoblot Analysis}

ccRCC samples of various grades and adjacent nontumoral kidney tissue were rapidly homogenized and lysed in $1 \% \mathrm{SDS}, 1.0 \mathrm{mmol} / \mathrm{L}$ sodium vanadate, and $10 \mathrm{mmol} / \mathrm{L}$ Tris [ $\mathrm{pH}$ 7.4]); then protein was collected and the protein concentration was determined using BCA protein assay kits (Thermo Fisher Scientific, Cramlington, Northumberland, UK). Proteins $(25 \mu \mathrm{g})$ in sample buffer were separated by SDS-polyacrylamide gel electrophoresis and then transferred to a nitrocellulose membrane and immunoblotted as described previously. ${ }^{18}$ Membranes were rinsed for 30 minutes in buffer $(0.1 \%$ Tween 20 in Tris-buffered saline) and then incubated with mouse anti-TNFR1 (1:250), anti-TNFR2 (1:500), anti-ASK1-pThr845, anti-phospho-Etk ${ }^{\text {Tyr40 }}$, and antiphospho-VEGFR2 ${ }^{\text {pY1054-59 }}$ (1:1000 dilution), followed by anti-mouse or anti-rabbit IgG horseradish peroxidase conjugate and detected by enhanced chemiluminescence according to the manufacturer's instructions.

\section{RNA Extraction, cDNA Synthesis, and Expression Quantitative Real Time-PCR}

RNA was isolated from human nontumoral kidney and ccRCC tumor samples, which had been snap-frozen in liquid nitrogen, using an RNA PureLink kit (Invitrogen, Paisley, UK), as recommended by the manufacturer with the following modifications. The tissue samples were thawed in $1.5 \mathrm{ml}$ of TRI Reagent (Sigma-Aldrich, St. Louis, MO) and homogenized for three 20-second bursts, using a handheld homogenizer (Polytron). RNA was isolated according to the manufacturer's protocol, and an on-column DNase treatment was included. RNA quality for all samples was checked using the QIAxcel bioanalyzer, and only samples with undegraded RNA were used for cDNA synthesis. Twenty microliters of cDNA was synthesized from $1 \mu \mathrm{g}$ of total RNA, using a mixture of both oligo(dT) and random hexamer and the SuperScript III First-Strand cDNA Synthesis Kit (Invitrogen). Quantitative real-time (qRT)-PCR of housekeeping genes was initially performed using $4 \mu \mathrm{l}$ of 1:20 prediluted cDNA in a 20- $\mu$ l reaction and TaqMan Gene Expression Assays specific for RPLPO and TBP. We obtained similar data by normalizing against either of these housekeeping genes individually or when a normalization factor was obtained using the geNorm algorithm (http://medgen.ugent. 
be/ jvdesomp/genorm/\#PrimerDesign, last accessed June 8, 2010) (data not included).

qRT-PCR was performed in triplicate on the same diluted cDNA samples for TNFR2 and the housekeeping genes using validated TaqMan Gene Expression Assay primers (Applied Biosystems, Foster City, CA). After normalization against RPLPO, statistical analysis for qRTPCR was performed using the one-way analysis of variance and a Bonferroni test, and $P<0.05$ was considered statistically significant.

\section{Terminal Deoxynucleotidyl Transferase-Mediated dUTP Nick End-Labeling Assay}

Apoptosis on paraffin wax sections was determined by the terminal deoxynucleotidyl transferase-mediated dUTP nick-end labeling (TUNEL) method as detailed in

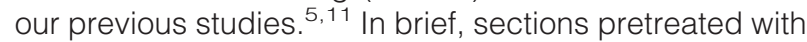
protease were nicked and labeled with TUNEL label mix and TdT enzyme according to the manufacturer's instructions (Roche Diagnostics, Burgess, West Sussex, UK) and examined using a confocal laser scanning microscope at $\times 40$ to $\times 63$.

\section{Immunofluorescence Analysis}

For quantification of positive normal TECs and mTECs, 19 randomized sections of nontumoral kidney and of the individual ccRCC grade samples were viewed under fluorescent illumination on a Nikon Optiphot Microscope (Nikon UK Ltd., Kingston on Thames, Surrey, UK) using a $\times 40 / 0.85$ NA dry objective by two independent observers blinded to the treatments. To avoid bias, cell count was standardized using the microscope stage. A beginning point was chosen at random and the next point was obtained by moving in a grid pattern across the section. This resulted in data from 10 unbiased and representative fields per section. Because the degree of immunofluorescence staining did not vary widely among samples, the intensity of staining was graded as either $(-)$ to $(+)$. Background fluorescence was determined for sections stained with preimmune serum or with secondary antibody alone. The numbers of cells positive for TUNEL or H3 $3^{\text {phospho-S10 }}$ (presented as apoptotic index or proliferative index) or $\mathrm{NF}-\kappa \mathrm{B}^{\mathrm{P}-\mathrm{S} 276-\mathrm{p} 65}$ were counted in a similar way on nontumoral kidney and ccRCCs from six different organ cultures from each of the four treatments. Likewise the numbers of positive cells for VEGFR2 ${ }^{\text {pY1054-59 }}$ or Etk ${ }^{\text {PTyr4O }}$ were quantified on cultures pretreated with VEGFR2-selective tyrosine kinase inhibitor.

\section{Statistical Analysis}

The results are presented as means \pm SEM. Differences between treatments was analyzed using an unpaired twotailed Student's $t$-test and analysis of variance. When statistically significant differences were found $\left({ }^{\star} P<0.001\right.$ or $\left.{ }^{\star \star} P<0.05\right)$, post hoc comparisons were performed using a Bonferroni comparison test in GraphPad Prism.

\section{Results}

\section{TNFR2 Is Induced and Up-Regulated in mTECs}

We first identified both normal TECs and mTECs in human renal biopsy samples by means of immunofluorescence staining for standardized markers. Nontumoral kidney showed a marked signal for RCC-Ma in the proximal tubules that was absent in distal tubules, glomeruli, and stromal cells. CD10 was detected in proximal tubules and in glomeruli, vimentin was observed in all cell types, and pan-CK was detected in all tubules, with a decrease or absence of staining seen in high-grade tumors. The pattern and staining intensity of the markers presented in Table 1 are consistent with previous studies $^{15,19-23}$ We then examined the expression of TNFR1 and TNFR2 in normal and ccRCC specimens. As we had reported previously ${ }^{5}$ normal (nontumoral and time 0 biopsy samples from kidneys harvested for transplantation), kidney showed marked expression for TNFR1 in glomeruli and vascular endothelial cells (VECs). whereas TNFR2 was detected in VECs at low levels and in leukocytic mononuclear cells (MNCs) (Figure 1A). Both TNFRs were rarely detected in normal TECs. Fuhrman grade 1 samples (Figure 1B, Supplemental Figure 1, A-D, see http://ajp.amjpathol.org) showed tumor cells with small round nuclei with inconspicuous or absent nucleoli. Parallel sections revealed a pattern of immunostaining similar to that of nontumoral kidney with a marked TNFR1

Table 1. Quantification of ccRCC-Associated Immunomarkers in Nontumoral Kidney and in ccRCC Tissue

\begin{tabular}{|c|c|c|}
\hline Immunomarkers & nTEC & mTEC \\
\hline \multicolumn{3}{|l|}{ Nontumoral kidney } \\
\hline RCC-Ma & $8.2 \pm 1.2$ & \\
\hline CD10 & $8.3 \pm 1.5$ & \\
\hline Vimentin & $2.5 \pm 1.0$ & \\
\hline Pan-cytokeratin & $9.1 \pm 0.5$ & \\
\hline \multicolumn{3}{|l|}{ ccRCC grades } \\
\hline \multicolumn{3}{|l|}{ Fuhrman grade 1} \\
\hline RCC-Ma & & $8.3 \pm 0.7$ \\
\hline CD10 & & $8.4 \pm 1.0$ \\
\hline Vimentin & & $8.1 \pm 1.2$ \\
\hline Pan-cytokeratin & & $7.5 \pm 1.4$ \\
\hline \multicolumn{3}{|l|}{ Fuhrman grade 2} \\
\hline RCC-Ma & & $8.1 \pm 1.4$ \\
\hline CD10 & & $8.6 \pm 1.1$ \\
\hline Vimentin & & $8.2 \pm 1.3$ \\
\hline Pan-cytokeratin & & $6.9 \pm 2.1$ \\
\hline \multicolumn{3}{|l|}{ Fuhrman grade 3} \\
\hline RCC-Ma & & $8.2 \pm 1.2$ \\
\hline CD10 & & $6.7 \pm 1.6$ \\
\hline Vimentin & & $8.4 \pm 1.3$ \\
\hline Pan-cytokeratin & & $2.3 \pm 0.9$ \\
\hline \multicolumn{3}{|l|}{ Fuhrman grade 4} \\
\hline RCC-Ma & & $8.4 \pm 1.1$ \\
\hline CD10 & & $8.3 \pm 1.1$ \\
\hline Vimentin & & $8.3 \pm 1.1$ \\
\hline Pan-cytokeratin & & $2.4 \pm 0.8$ \\
\hline
\end{tabular}

A marked diffuse signal for RCC-Ma, CD10, and pan-cytokeratin was detected in normal TECs (nTECs). All cCRCC tissue showed a similar pattern and intensity of staining for RCC-Ma and CD10 in mTECs with a statistically significant increase in vimentin and a decrease pan-cytokeratin expression in severe tumors. Results are means $\pm \mathrm{SEM} ; n=19$ per group with similar results. Significant differences between nTEC and mTEC are shown in bold $(P<0.01)$. 

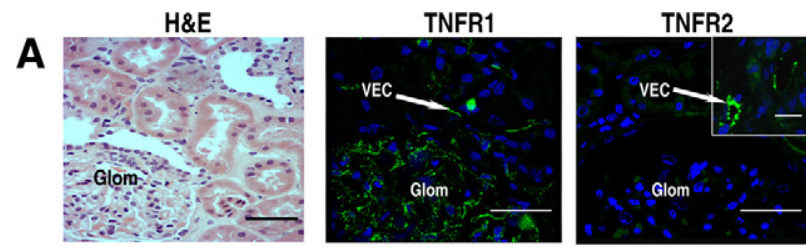

\section{B}
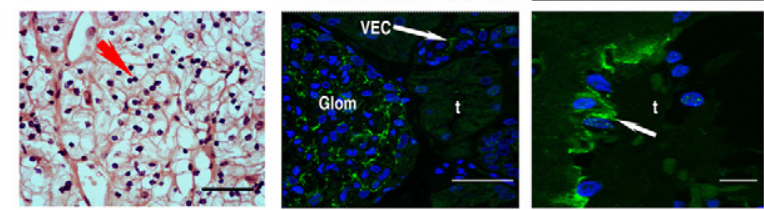

C
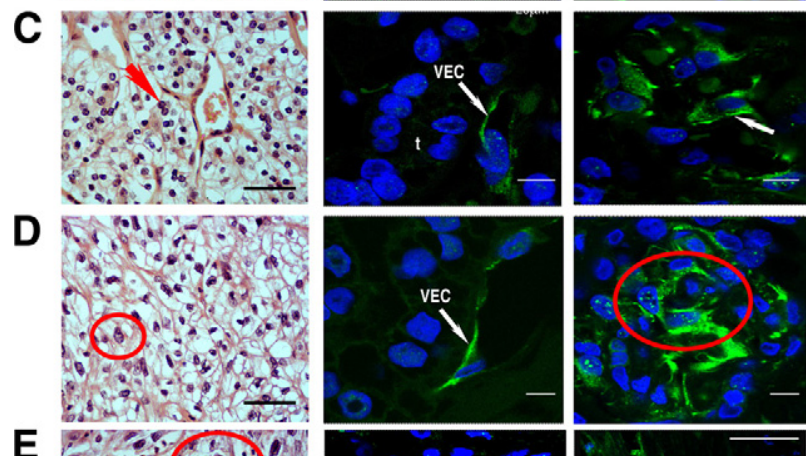

$\mathbf{E}$
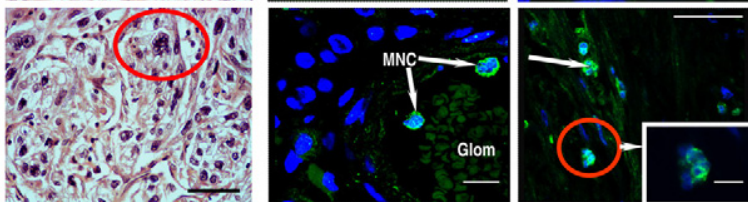

Figure 1. H\&E and immunofluorescent staining of TNFRs in nontumoral kidney (A) and in the different grades of ccRCC samples: Fuhrman grade 1 (B); Fuhrman grade 2 (C); Fuhrman grade 3 (D); and Fuhrman grade 4 (E) (red circles indicate pleiotropic nuclei in grade 3 tumor; inset show a high-power image of a TNFR2-positive multilobed mTECs in grade 4 tumor). Arrows show cytoplasmic pattern of TNFR2 expression in Malignant Tubular Epithelial Cells (mTEC; t). Representative immunohistochemical staining. Glom, glomerular. Scale bars: $100 \mu \mathrm{m}$ (H\&E); $20 \mu \mathrm{m}(\mathbf{A}$ and B) $50 \mu \mathrm{m}(\mathbf{C}-\mathbf{E})$

signal seen in glomerular cells and VECs but rarely in normal TECs or mTECs. In contrast, TNFR2 was moderately expressed in VECs and MNCs, and weakly in mTECs, which were also positive for RCC-Ma and CD10. The subcellular expression pattern for both receptors was mainly diffusely cytoplasmic staining

Fuhrman grade 2 samples (Figure 1C, Supplemental Figure 2, A-D, see http://ajp.amjpathol.org) showed tumor cells with medium-sized nuclei with slightly irregular contours and often with small but distinct nucleoli. Immunostaining of sequential sections showed diminished TNFR1 expression in glomerular cells and VECs, both negative for RCC-Ma but positive for CD10. TNFR1 was rarely detected in either normal TECs or in mTECs. A noticeable increase in TNFR2 expression was observed in mTECs, also reactive for RCC-Ma and CD10. TNFR2 showed mainly a cytoplasmic pattern of staining, whereas RCC-Ma and CD10 showed membranous patterns of staining.

Fuhrman grade 3 samples (Figure 1D, Supplemental Figure 3, A-D, see http://ajp.amjpathol.org) showed irregular larger nuclei with prominent nucleoli. Immunostaining of sequential sections showed diminished expression of TNFR1 in VECs but a marked signal in MNCs, negative for RCC-Ma or CD10. A striking increase in TNFR2 ex-
Table 2. Quantification of TNFR Expression in Nontumoral Kidney and in ccRCC Tissue

\begin{tabular}{|c|c|c|}
\hline TNFR & nTEC & mTEC \\
\hline \multicolumn{3}{|c|}{ Nontumoral kidney } \\
\hline TNFR1 & $0.5 \pm 0.5$ & \\
\hline TNFR2 & $0.4 \pm 0.5$ & \\
\hline \multicolumn{3}{|c|}{ Fuhrman grade 1} \\
\hline TNFR1 & & $0.5 \pm 0.5$ \\
\hline TNFR2 & & $3.3+/-1.1$ \\
\hline \multicolumn{3}{|c|}{ Fuhrman grade 2} \\
\hline TNFR1 & & $0.4 \pm 0.4$ \\
\hline TNFR2 & & $8.0 \pm 1.1$ \\
\hline \multicolumn{3}{|c|}{ Fuhrman grade 3} \\
\hline TNFR1 & & $0.4 \pm 0.5$ \\
\hline TNFR2 & & $10.4 \pm 1.1$ \\
\hline \multicolumn{3}{|c|}{ Fuhrman grade 4} \\
\hline TNFR1 & & $0.4 \pm 0.5$ \\
\hline TNFR2 & & $12.0 \pm 1.2$ \\
\hline
\end{tabular}

Nontumoral kidney (control) showed negligible TNFR1 and TNFR2 expression in normal tubular epithelial cells (nTECs). In contrast, a significant up-regulation in TNFR2 is observed in mTECs, which increases with tumor grade. Results are means $\pm \mathrm{SEM} ; n=19$ per group with similar results. Significant differences comparing TNFR2 expression in mTEC among different RCC grades and control are marked in bold $(P<0.001)$

pression was detected in infiltrating MNCs and in mTECs, also positive for RCC-Ma or CD10.

Fuhrman grade 4 samples (Figure 1E, Supplemental Figure 4, A-D, see http://ajp.amjpathol.org) showed some tumor cells with multilobed nuclei in addition to cells with grade 3 nuclei. Immunostaining of sequential sections showed rare TNFR1 expression in glomerular cells and VECs, but a marked signal in MNCs, which were negative for RCC-Ma or CD10. Compared with Fuhrman grade 2 and 3 samples, TNFR2 was drastically elevated in mTECs, of which the RCC-Ma- and CD10-reactive tumor cells were either isolated or appeared in small clusters, some containing a multilobed nuclei. A summary of TNFR expression in nontumoral kidney and different grades of cCRCC is shown in Table 2. We next performed qRT-PCR to determine the expression profile of the TNFR2 transcript in normal kidney and different grades of ccRCC. Our results demonstrate up-regulation in the expression of TNFR2 in ccRCC grade 2, 3 , and 4 samples compared with the normal kidney samples (Figure 2). These data show that TNFR2 expression is modulated transcriptionally. No differences were seen in the expression of TNFR1 mRNA between normal kidney and ccRCC (data not shown). Collectively, these data indicate that mTECs express TNFR2 and that expression increases with higher grades of malignancy.

\section{wtTNF or R1-TNF Induces Activation of ASK1, Whereas wtTNF or R2-TNF Induces Activation of Phospho-Etk and -VEGFR2 in mTECs in cCRCC Organ Cultures}

To examine the functions of TNFR expression, we treated samples of human kidney biopsies with wtTNF or with receptor-selective TNF muteins in short-term organ culture and analyzed responses of individual cells by immunostaining. Cultures of nontumoral kidney showed ex- 


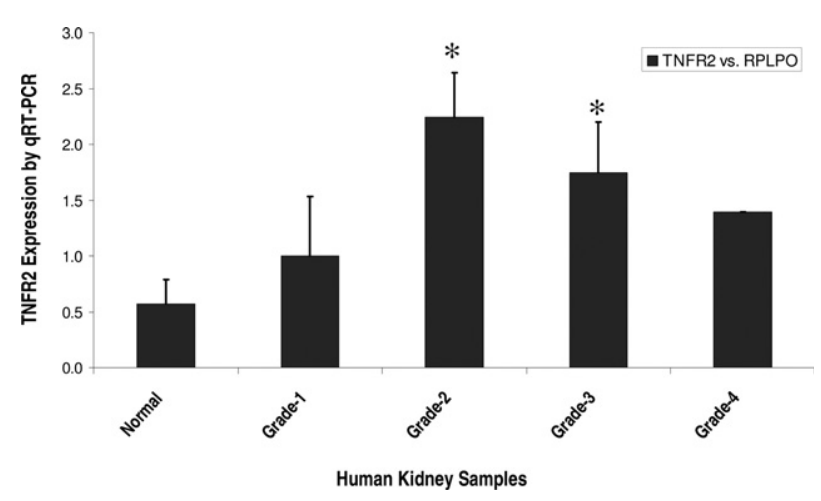

Figure 2. Quantitative RT-PCR analysis of the TNFR2 in nontumoral kidney and in the different grades of ccRCC samples. There is a statistically significant higher level of TNFR2 transcript in grade 2 to 4 samples compared with nontumoral kidney samples $\left({ }^{*} P<0.05\right)$. All qRT-PCR data were from experiments performed in triplicate and were normalized versus the RPLPO housekeeping gene (similar data were obtained when normalized against TBP; data not included). Results are average with SD and are representative of at least two independent experiments, with the exception of grade 4 ccRCC in which RNA degradation meant that only one sample was suitable for analysis.

pression of TNFRs and phosphorylation of ASK1 and Etk by wtTNF and TNFR-specific muteins as reported previously. ${ }^{11}$ A signal for phospho-VEGFR2 ${ }^{\text {pY1054-105 }}$ and VEGF was detectable in glomerular and peritubular capillaries but not in TECs (data not shown). Untreated cCRCC organ cultures (Figure 3A) showed TNFR1 in glomerular cells and VECs, where it costained with the inactive form of ASK1-pSer967 but not with the active form of ASK1-pThr845. TNFR2 was moderately present in VECs and in MNCs but not in normal TECs with a weak signal observed in mTECs. Phospho-Etk ${ }^{\text {Tyr40 }}$ expression

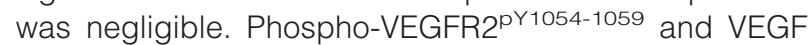
were strongly detected in glomerular cells and VECs and rarely in mTECs (data not shown). We also analyzed TNFRs and their downstream signaling molecules in cCRCC tissue and adjacent normal tissue by immunoblot analysis. Consistent with the immunohistochemistry results, we found that TNFR2, phospho-VEGFR2 ${ }^{\text {pY1054-1059, and }}$ phospho-Etk ${ }^{\mathrm{pTyr} 40}$ expression was higher in ccRCC than in adjacent normal kidney tissue (Figure 3B). Comparable levels of TNFR1 were expressed in both normal kidney and in cCRCC. Activated ASK1 was not detected in either sample (data not shown). These findings are consistent with our analysis by immunostaining of sections. Table 3 summarizes the differences in TNFRs expression and their associated molecules in mTECs in cCRCC organ cultures.

wtTNF-treated cultures (Figure 3C) showed a marked increase in TNFR1 that colocalized with ASK1-pThr845 in mTECs. TNFR2 was also dramatically increased in mTECs where it colocalized with phospho-Etk ${ }^{\text {PTyr4O }}$. R2TNF-treated cultures (Figure 3D) did not induce TNFR1 or ASK1-pThr845 expression in mTECs but showed a marked increase in TNFR2 and its colocalization with phospho-Etk ${ }^{\text {Tyr4O }}$. In contrast, wtTNF- and R2-TNFtreated cultures showed noticeably increased expression of phospho-VEGFR2 ${ }^{\text {PY1054-1059 }}$ but not of VEGF, where it costained with TNFR2 or phospho-Etk ${ }^{\text {PTyr4O }}$ in mTECs. R1-TNF-treated cultures (Figure 3E) showed a pattern and intensity of staining for TNFR1 and ASK1-pThr845 similar to those for wtTNF-treated cultures, but R1-TNFtreated cultures did not induce TNFR2 or phospho-Etk ${ }^{\text {PTyr4O }}$ in mTECs. R1-TNF-treated cultures showed a pattern and intensity of staining for VEGF and phosphoVEGFR2 ${ }^{\mathrm{pY} 1054-1059}$ similar to those of untreated cultures (data not shown). Nontumoral kidney showed patterns and intensity of immunostaining similar to those of untreated cultures and as previously reported. ${ }^{10,11}$ Collectively, the data demonstrate that wtTNF or R1-TNF induces activation of ASK1, whereas wtTNF or R2-TNF induces activation of phospho-Etk ${ }^{\text {Tyr } 40}$ and phosphoVEGFR2 ${ }^{\text {pY1054-1059 }}$ without increasing expression of VEGF in $\mathrm{MTEC}$.

\section{wtTNF or R1-TNF Promotes Apoptosis and NF- $\kappa B$ Activation, Whereas wtTNF or R2-TNF Promotes Cell Cycle Entry and NF- $\mathrm{B}$ Activation in $m$ TECs in cCRCC Organ Cultures}

Having established some of the proximal signaling events activated by both TNFR1 and TNFR2 in mTECs, we then analyzed more downstream cellular responses. As shown in Figure 4A, untreated cultures without TNF showed an occasional signal for cleaved active caspase$3^{\text {Asp175 }}$ and TUNEL in mTECs, with expression detected in VECs and in MNCs. In contrast, increased expression was observed in mTECs in R1-TNF- or wtTNF-treated cultures. The pattern and intensity of immunostaining in R2-TNF-treated cultures was comparable to those in untreated cultures. A statistically significant difference in the level of TUNEL-positive nuclei in mTECs was demonstrated among cultures, with the highest level of staining evident in R1-TNF- or wtTNF-treated cultures compared with untreated and R2-TNF-treated cultures $(P<0.0001)$. An apoptotic index was derived from the average number of TUNEL-positive cells (Figure 4B), consistent with this conclusion.

Because TNF has been positively correlated with the invasive phenotype of $\mathrm{RCC},{ }^{24}$ we investigated the relationship between TNF and expression of cell cycle markers such as $\mathrm{H}^{\text {phospho-S10 }}$ and $\mathrm{Ki}-67$ in our ccRCC organ cultures. Untreated or R1-TNF-treated cultures showed a negligible level of nuclear $\mathrm{H}^{\text {phospho-S10 }}$ expression in mTECs, whereas wtTNF- or R2-TNF-treated cultures showed a strong signal (Figure 5A). A statistically significant difference in nuclear $\mathrm{H}^{\text {phospho-S10 }}$ expression was evident between untreated and R2-TNF- or wtTNFtreated cultures $(P<0.001)$ and between R2-TNF- and wtTNF-treated cultures $(P<0.05)$ (Figure 5B, Table 3).

Untreated cultures showed negligible NF- $\mathrm{B}^{\mathrm{P}-\mathrm{S} 276-\mathrm{p} 65}$ expression. In contrast, R1-TNF-treated cultures showed increased expression in mTECs with a higher level of expression in wtTNF-treated cultures and even higher level in R2-TNF-treated cultures (Figure 6A). Statistically significant differences $(P<0.0001)$ were detected between cultures (Figure 6B, Table 3). Differences in expression were also detected between untreated and R1-TNF-treated cultures $(P<0.05)$ but not between 
A

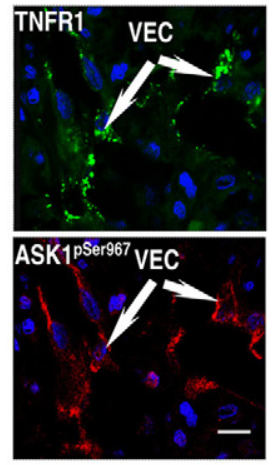

C
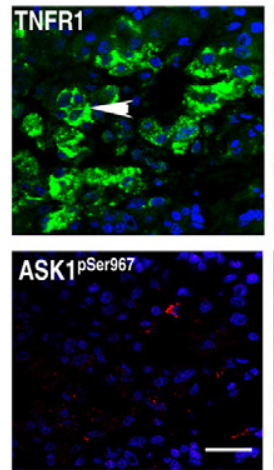

D
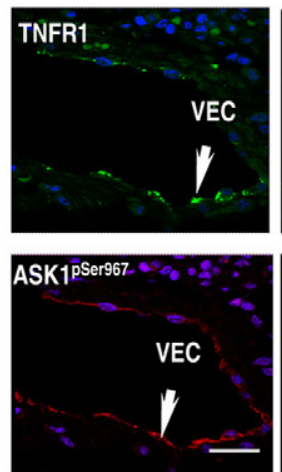

E
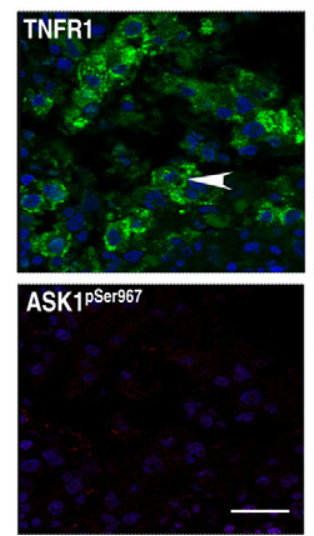
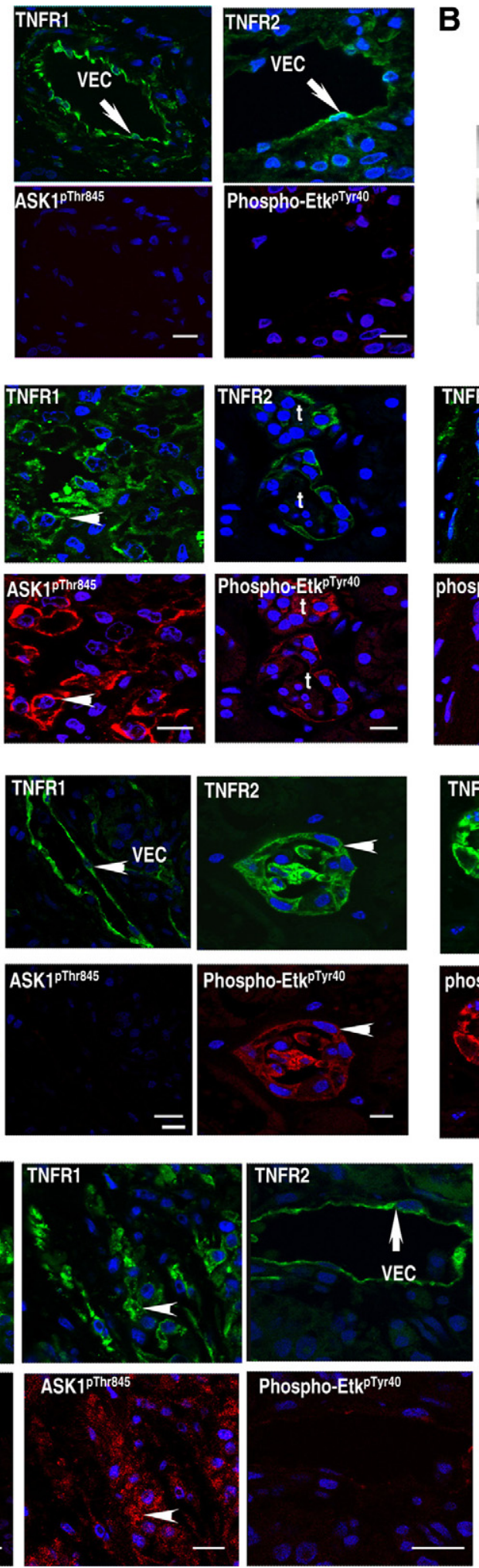

B

\section{Normal kidney ccRCC}
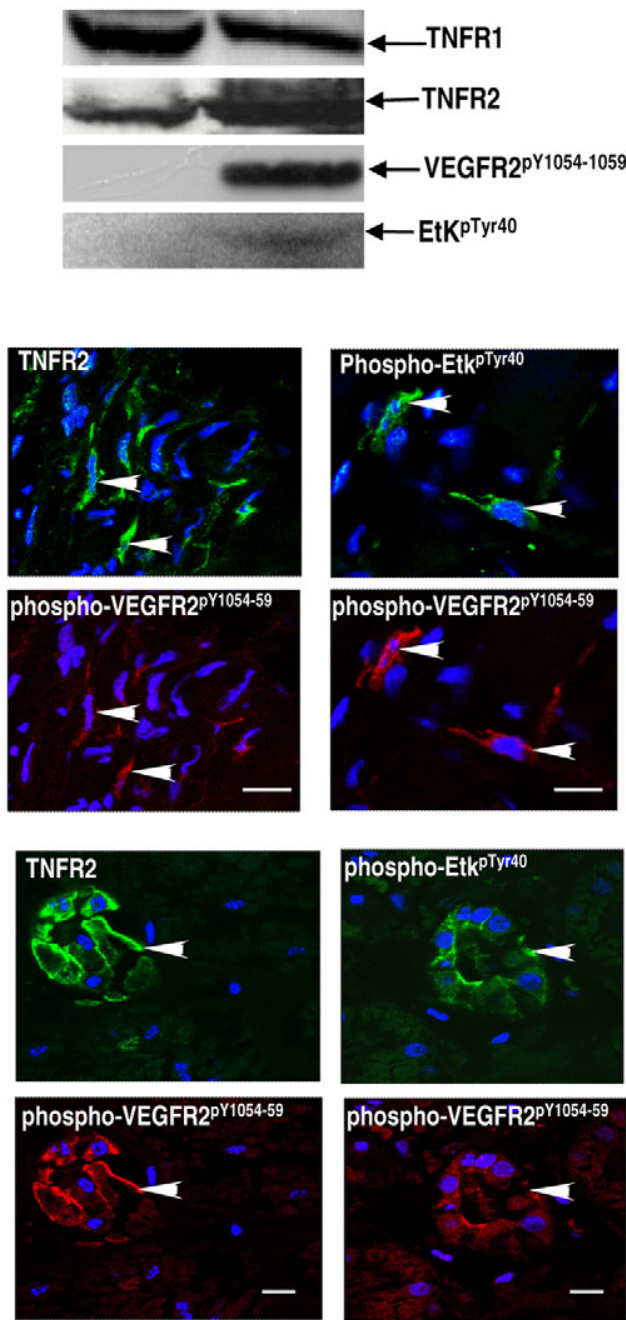

Figure 3. Coimmunofluorescent staining of TNFR1 and ASK1-pSer967 or -pThr845 or TNFR2, and phospho-Etk ${ }^{\text {pTry } 40}$ in ccRCC organ cultures. A: Untreated cultures show a marked colocalization of TNFR1 and ASK1-pSer967 and of TNFR2 in VEC, with negligible phospho-Etk ${ }^{\text {pTry }^{40}}$. B: Immunoblot analysis of TNFRs and their downstream signaling molecules in cCRCC tissue and adjacent normal tissue shows a higher expression of phospho-VEGFR2 $2^{\text {pY1054-1059 }}$ and TNFR2 in ccRCC compared with adjacent normal kidney tissue. TNFR1 is expressed in normal kidney and ccRCC. A weak signal for phospho-Etk ${ }^{\text {Tyr } 40}$ is detected in ccRCC. ASK1-pThr845 is not detected in normal kidney or ccRCC. C: wtTNF-treated cultures show a marked colocalization of TNFR1 and ASK1-pThr845 (arrowheads) or TNFR2 and phosphoEtk $^{\mathrm{p}^{\mathrm{Tyr} 40}}$ in mTECs; malignant tubular epithelial cells $(\mathrm{t})$. Colocalization of phospho-VEGFR2 ${ }^{\text {PY1054-1059 and TNFR2 or phospho-Etk }}{ }^{\mathrm{p}^{T r y} 40}$ is evident in mTECs (arrowheads). D: R2-TNF-treated cultures showed a pattern of staining similar to that of wtTNF but did not induce ASK1-pThr845 expression. E: R1-TNF-treated cultures show up-regulation and colocalization of TNFR1 and ASK1-pThr845 but not phospho-Etk in mTECs. Representative images from one of six different ccRCC organ cultures, each of which gave similar results. Nuclei counterstained with To-PRO-3' iodide. Repeated measurements yielded identical results. Scale Bar; $50 \mu \mathrm{M}$. 
Table 3. Quantification of TNFR Expression and Their Associated Molecules in mTECs in ccRCC Organ Cultures Incubated Either in Media Alone (Untreated without TNF) or with wtTNF or R1-TNF- or R2-TNF-Mutein

\begin{tabular}{|c|c|c|c|c|c|c|c|c|c|}
\hline Treatment & TNFR1 & TNFR2 & $\begin{array}{c}\text { ASK1- } \\
\text { pSer967 }\end{array}$ & $\begin{array}{l}\text { ASK1- } \\
\text { pThr845 }\end{array}$ & Etkp & VEGF & pVEGFR2 & $\mathrm{pH} 3$ & $N F-\kappa B^{S-276 p 65}$ \\
\hline Untreated & $0.5 \pm 0.5$ & $0.3+0.1$ & $0.5+0.5$ & $0.1+0.1$ & $0.5+0.5$ & $0.1+0.0$ & $0.2+0.1$ & $0.5 \pm 0.5$ & $0.7 \pm 0.2$ \\
\hline R1-TNF & $7.1 \pm 1.6$ & $0.2 \pm 0.1$ & $0.5 \pm 0.5$ & $7.9 \pm 0.2$ & $0.5 \pm 0.4$ & $0.1 \pm 0.0$ & $0.5 \pm 0.5$ & $0.5 \pm 0.5$ & $3.5 \pm 0.2$ \\
\hline R2-TNF & $0.5 \pm 0.5$ & $8.3 \pm 1.7$ & $0.5 \pm 0.5$ & $0.1 \pm 0.1$ & $7.1 \pm 1.7$ & $0.1 \pm 0.3$ & $8.7 \pm 1.6$ & $7.7+/ 1.4$ & 8.0. \pm 1.4 \\
\hline wtTNF & $8.6 \pm 0.9$ & $7.7 \pm 1.6$ & $0.4 \pm 0.2$ & $7.6 \pm 2.1$ & $8.0 \pm 1.6$ & $0.3 \pm 0.2$ & $8.9 \pm 1.5$ & $4.2 \pm 1.7$ & $4.7 \pm 1.7$ \\
\hline
\end{tabular}

Results are means $\pm \mathrm{SEM} ; n=6$ per group with similar results. Significant differences comparing untreated versus TNF-treated cultures are marked in bold $(P<0.001)$

R1-TNF- and wtTNF-treated cultures. Expression of nuclear $\mathrm{H} 3^{\text {phospho-S10 }}$ and NF- $\mathrm{B}^{\mathrm{P}-\mathrm{S} 276-\mathrm{p} 65}$ in mTECs was confirmed on parallel sections coimmunostained with RCC markers (RCC-Ma and CD10; Supplemental Figures 5 and 6, see http://ajp.amjpathol.org).

Superficially, these data seem to pose a paradox. If RCC cells produce TNF and if TNF, acting through TNFR1 and TNFR2, induces TNFR1 and TNFR2, respectively, why do RCC cells express TNFR2 but not TNFR1?

A
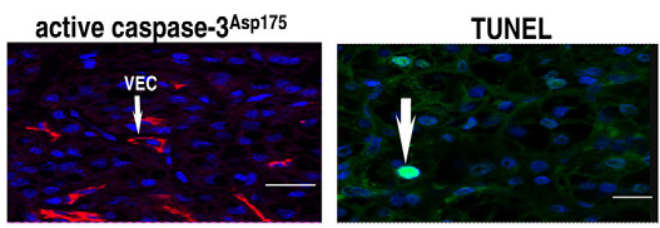

wtTNF
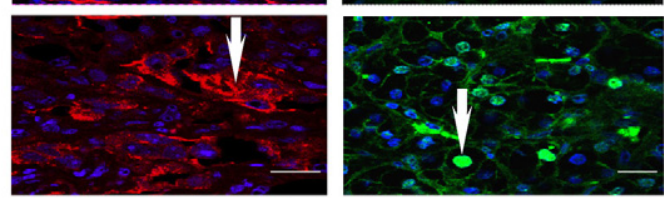

R1-TNF
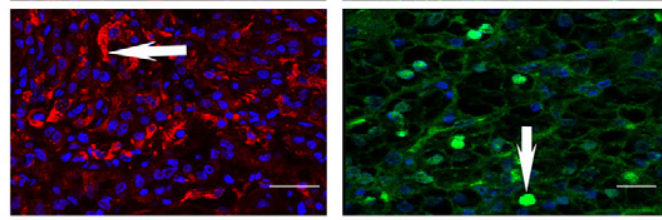

R2-TNF
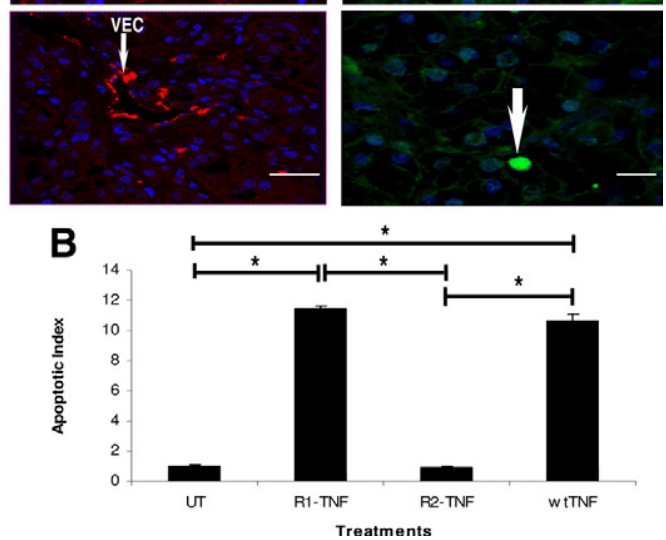

Figure 4. A: Active cleaved caspase- $3^{\mathrm{Asp} 175}$ and TUNEL expression in ccRCC organ cultures. Untreated cultures (without TNF) show a rare signal for active caspase-3 in a VEC (small arrow) and TUNEL (large arrow) in a mTEC. In contrast, wtTNF- and R1-TNF-treated cultures show a strong signal for active caspase-3 and TUNEL in mTECs (arrows). R2-TNF-treated cultures show a pattern of staining similar to that of untreated cultures. Nuclei were counterstained with To-PRO-3' iodide. B: Analysis of TUNEL-reactive mTECs by paired Student's $t$-test; data are presented as apoptotic index. Untreated (UT) or R2-TNF versus R1-TNF or wtTNF: ${ }^{*} P<0.0001$. Data are means \pm SEM; $n=$ 6 per group with similar results. Scale bars: $200 \mu \mathrm{m}$ (caspase-3); $20 \mu \mathrm{m}$ (TUNEL).
A possible solution is that TNFR2 signaling is activated at a lower concentration of TNF than is TNFR1 signaling. To test this possibility, we treated parallel ccRCC organ cultures for 3 hours with 0, 0.1, 1, 3, and $10 \mathrm{ng} / \mathrm{ml}$ wtTNF. At a dose of $10 \mathrm{ng} / \mathrm{ml}$, wtTNF induced a marked increase in expression of both TNFR1 and TNFR2 in mTECs. However, TNFR1 was not induced in mTECs at lower doses of $0.1,1$, and $3 \mathrm{ng} / \mathrm{ml}$ wtTNF, whereas expression of TNFR2 was increased after treatment with $3 \mathrm{ng} / \mathrm{ml}$ but not 1 or $0.1 \mathrm{ng} / \mathrm{ml}$ wtTNF (data not shown). Thus, the concentration of TNF in CCRCC may be insufficient to induce TNFR1. A differential effect on caspase activation and cell cycle entry was also observed at different TNF doses.

A
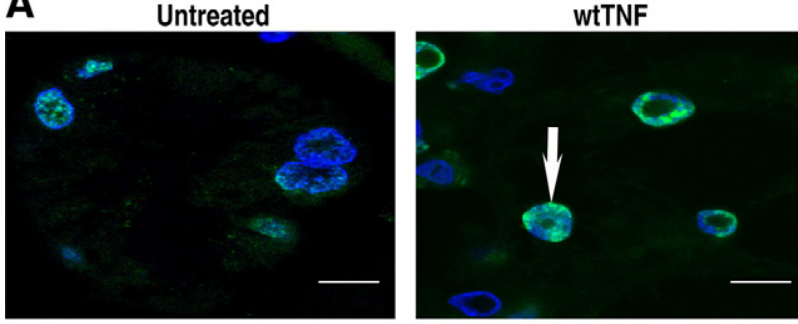

R1-TNF

R2-TNF
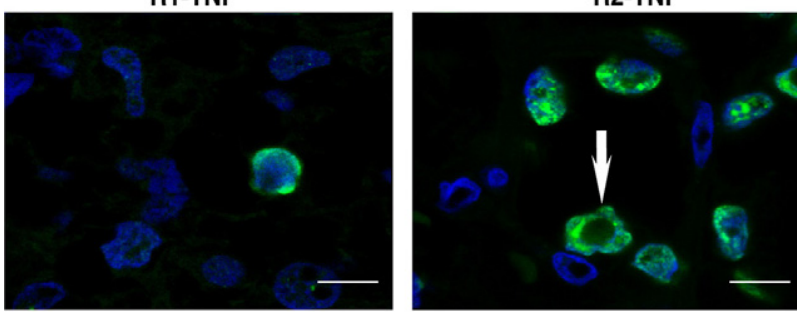

B

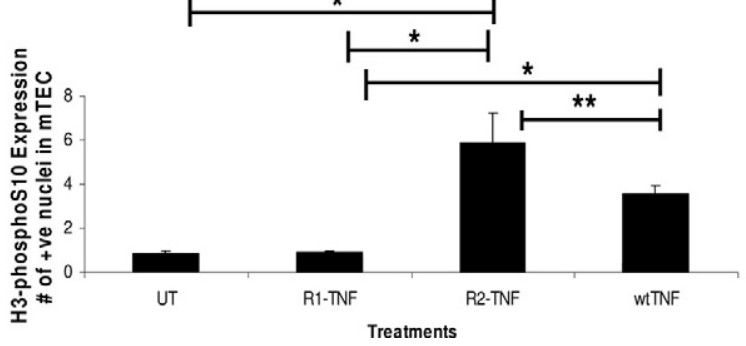

Figure 5. A: Nuclear $\mathrm{H} 3^{\text {phospho- } \mathrm{S} 10}$ expression in ccRCC organ cultures. Untreated cultures (without TNF) and R1-TNF-treated cultures show a rare signal for $\mathrm{H} 3^{\text {phospho-s10 }}$ in mTECs. In contrast, wtTNF- and R2-TNF-treated cultures show increased expression for $\mathrm{H}^{\text {phosphosio }}$ in mTECs (arrows). Nuclei counterstained with To-PRO-3' iodide. B: Analysis by paired Student's $t$-test. Untreated (UT) or R1-TNF versus R2-TNF or wtTNF: ${ }^{*} P<0.0001$; R2-TNF versus wtTNF: ${ }^{* *} P<0.05$. Data are means \pm SEM; $n=6$ per group with similar results. Scale bars $=50 \mu \mathrm{m}$. 
A

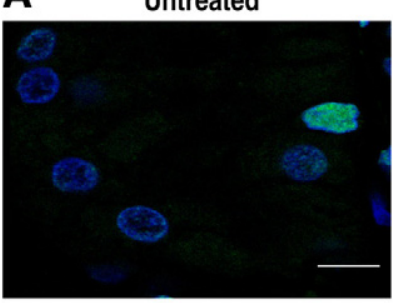

R1-TNF

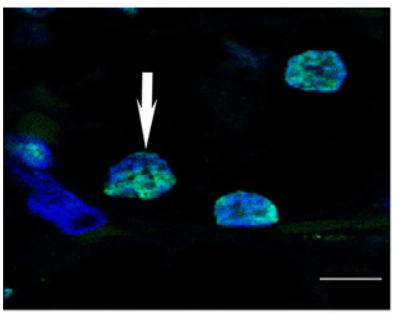

B

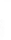

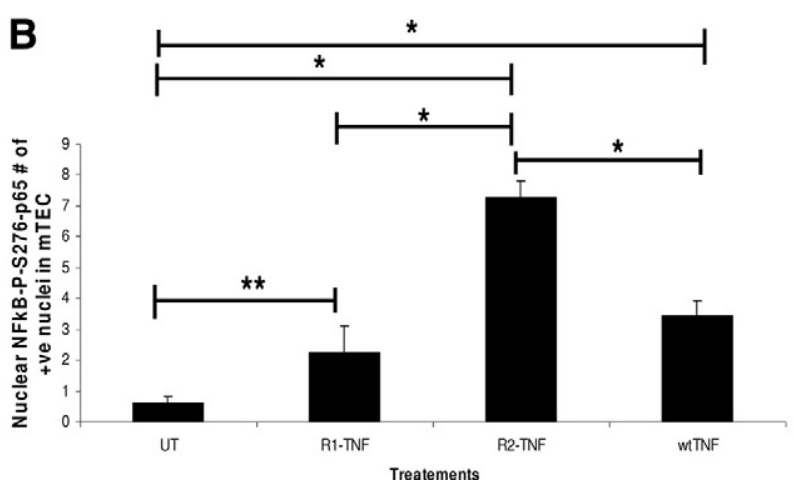

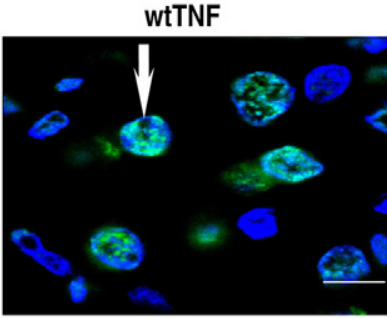

R2-TNF

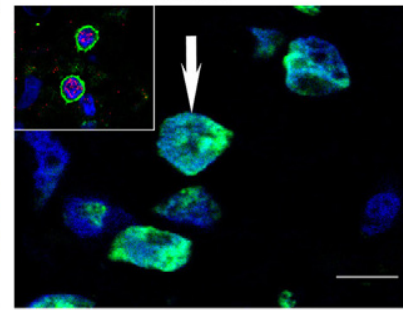

Figure 6. A: $\mathrm{NF}-\kappa \mathrm{B}^{\mathrm{P}-\mathrm{S} 276-\mathrm{p} 65}$ expression in ccRCC organ cultures. Untreated cultures (without TNF) show rare NF- $\kappa \mathrm{B}^{\mathrm{P}-\mathrm{S} 276-\mathrm{p} 65}$ expression in mTECs. In contrast, wtTNF- and R2-TNF-treated cultures show marked expression in mTECs (arrows). A positive signal but at a much lower level is detected in R1-TNF-treated cultures. Dual immunostaining show colocalization of ICAM-1 (cytoplasm; green) and NF- $\kappa \mathrm{B}^{\mathrm{P}-\mathrm{S} 276-\mathrm{p}^{65}}$ (nuclei; red) in some tumor cells. Nuclei counterstained with To-PRO-3' iodide. B: Analysis by paired Student's $t$-test. Untreated versus R2-TNF or wtTNF and R2-TNF versus wtTNF: ${ }^{*} P<0.001$; untreated versus R1-TNF: ${ }^{* *} P<0.05$. Data represent the means \pm SEM; $n=6$ per group with similar results. Scale bars $=50 \mu \mathrm{m}$.

Caspase activation was seen at $10 \mathrm{ng} / \mathrm{ml}$ but not at lower doses, whereas increased expression of $\mathrm{Ki}-67$ and $\mathrm{H} 3^{\text {phospho-S10 }}$ were seen at both 3 and $10 \mathrm{ng} / \mathrm{ml}$ wtTNF. These data support the interpretation that the levels of TNF made by RCC are capable of triggering TNFR2 but not TNFR1 signaling in an autocrine/paracrine manner.

We next determined whether activation of downstream signaling molecules precedes changes in protein expression of TNFRs, caspase activation, and cell cycle entry by treating ccRCC organ cultures with wtTNF or receptor-selective muteins at various time points (5 minutes, 30 minutes, 1 hour, 2 hours, and 4 hours). In ccRCC organ cultures, wtTNF and R1-TNF increased expression of ASK1-pThr845 at 2 and 4 hours, but not earlier time points. wtTNF and R2-TNF increased expression of Etk ${ }^{\mathrm{p}}$. Tyr40 and VEGFR2 ${ }^{\mathrm{pY} 1054-1059}$ at 2 and 4 hours, but not earlier time points (data not shown). The pattern of expression at these time points was similar to that seen in cultures treated for 3 hours (Figures 2, 4, and 5). A similar time course was seen for changes in the expression of TNFRs, with changes in TNFR expression at 2 and 4 hours being similar to those seen at 3 hours. Although caspase-3 activation was detectable at 2 hours in wtTNFand R1-TNF-treated ccRCC organ cultures, a marked increase in caspase-3 activation was not apparent until 4 hours, Increased $\mathrm{Ki}-67$ and $\mathrm{H} 3^{\text {phospho-S10 }}$ expression also only became apparent after 4 hours of treatment with wtTNF and R2-TNF. Because the kinetics of ASK1, Etk, and VEGFR2 phosphorylation are similar to those seen for increased TNFR expression, these kinases may not be involved in the signaling pathways leading to increased TNFR expression. In contrast, the kinetics of caspase activation and cell cycle entry are slower than those for ASK1, Etk, and VEGFR2 phosphorylation, consistent with a role for these kinases in signaling these events.

\section{wtTNF, VEGF, and R2-TNF Activate VEGFR2, Whereas wtTNF and R2-TNF (But Not VEGF) Induce Activation of Etk and Elevated Levels of

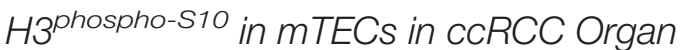

\section{Cultures}

Previous studies have shown that TNF induces a coordinated phosphorylation of Etk ${ }^{\text {PTyr40 }}$ and VEGFR2 ${ }^{\text {pY1054-1059, }}$ which is blocked by VEGFR2-specific inhibitors in endothelial cells. ${ }^{4}$ We therefore analyzed the effects of wtTNF-,
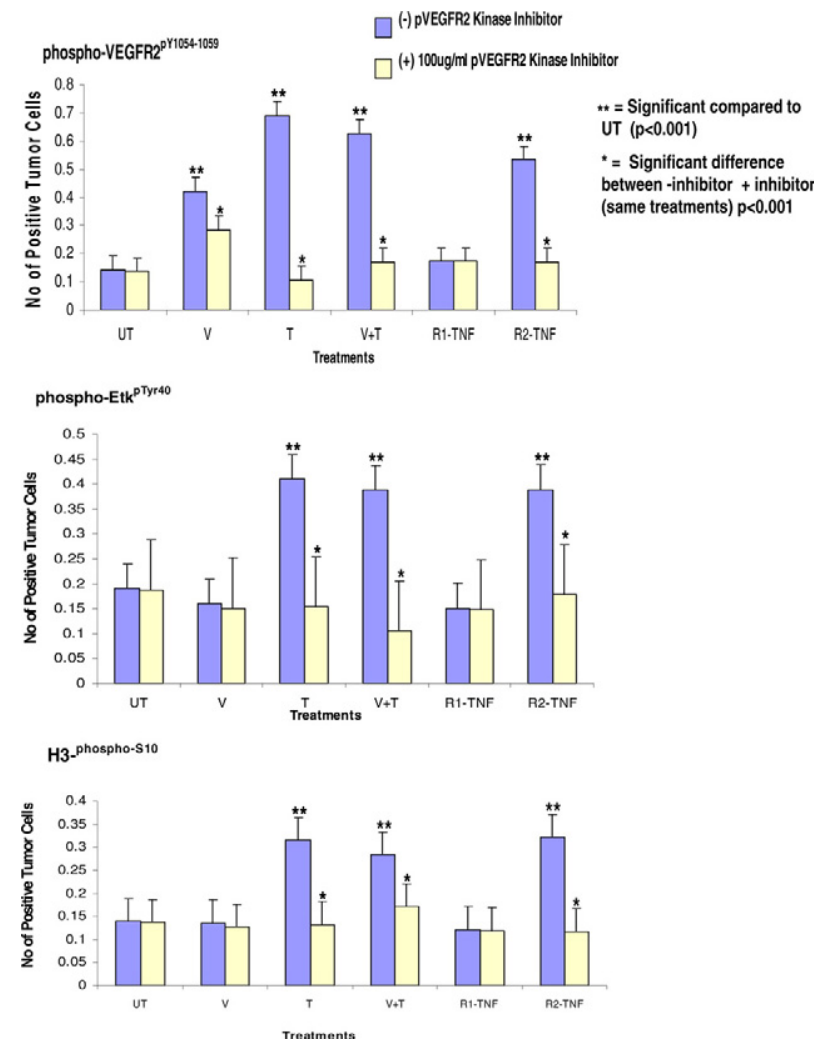

Figure 7. Expression of phospho-VEGFR2 ${ }^{\text {pY1054-1059, }}$, phospho-Etk ${ }^{\text {PTyr40 }}$, and $\mathrm{H} 3^{\text {phospho-S10 }}$ in malignant TEC in ccRCC organ cultures. Protein expression was analyzed by immunohistochemical analysis and quantified by analysis of variance followed by a Bonferroni comparison test. Each bar represents the mean \pm SEM of 10 fields of each group from six independent experiments. Significant compared with untreated (UT) $\left({ }^{* * *} P<0.001\right)$ Significant difference between without $(-)$ inhibitor and with $(+)$ inhibitor (same treatments; $\left.{ }^{*} P<0.001\right)$. V, VEGF; T, TNF. 
VEGF-, and TNFR-selective muteins on phosphorylation and activation of VEGFR2 and Etk in cCRCC organ cultures. We initially tested three different concentrations of SU1498, a VEGFR2-selective tyrosine kinase inhibitor, in our cCRCC organ cultures, and optimal inhibitory effects were detected with $100 \mu \mathrm{g} / \mathrm{ml}$, which was used in all subsequent experiments. As described before, wtTNF and R2-TNF induced a significant increase in the level of phospho-VEGFR2 ${ }^{\text {pY1054-1059 }}$ and of phospho-Etk ${ }^{\text {PTyr4O }}$ in mTECs. As described in endothelial cells, both signals are diminished in cultures pretreated with a VEGFR2specific tyrosine kinase inhibitor. On the other hand, VEGF, as expected, showed increase levels of phospho-

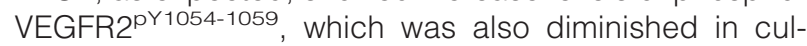
tures pretreated with the VEGFR2-specific tyrosine kinase inhibitor. However, in contrast to R2-TNF, VEGF did not induce activation of Etk (Figures 7 and $8, A$ and $B$ ), suggesting that Etk-VEGFR2 interactions are specific to TNF signaling. ${ }^{4}$ In contrast, untreated and R1-TNFtreated cultures showed only a basal level of phosphoVEGFR2 ${ }^{\text {PY1054-1059 }}$ and activation of Etk consistent with the fact that $\operatorname{Tyr}(\mathrm{P})-1054-1059$ is required for VEGFR2 autokinase. ${ }^{4}$ Similar results were observed in nontumoral kidney organ cultures but with a less drastic intensity and frequency of staining as shown in Supplemental Figure 7 (see http://ajp.amjpathol.org). Remarkably, treatments with wtTNF and R2-TNF but not with VEGF resulted in elevated expression of nuclear $H 3^{\text {phospho-s10 }}$ in mTECs (Figures 7 and 8C) and Ki-67 (data not shown).

Collectively these results provide direct evidence that TNFR2 growth effects in mTECs are mediated through its interactions with Etk and VEGFR2 and further indicate that TNFR2 and VEGFR2 signaling are distinct because only the former leads to activation of Etk and mTEC cycle entry in our organ culture system.

\section{Discussion}

Although TNF has been implicated in the pathogenesis of $\mathrm{RCC},{ }^{2,24-26}$ the mechanism and role of specific TNFRs in ccRCC has not been addressed previously. We have examined the expression of TNFRs in different grades of ccRCC and investigated the cellular responses triggered by TNFR ligation in ccRCC tissue using an organ culture model. ${ }^{11}$ To identify tumor cells we have used a panel of established immunomarkers in conjunction with comparative morphological studies. ${ }^{14,19,27}$ We report several new findings. i) In ccRCC tissue, TNFR2 increases in TECs with malignant grade, consistent with the observation that soluble (shed) TNFR2 plasma levels are significantly higher in patients with metastatic RCC and increase with tumor stage, ${ }^{28}$ but TNFR1 is only minimally expressed by mTECs regardless of grade. ii) In RCC organ culture TNFR1 ligation by TNF induces TNFR1 and TNFR2 ligation by TNF induces TNFR2, but TNFR2 responses occur at a lower concentration of TNF, consistent with the idea that TNF released by mTECs may selectively activate TNFR2 responses. iii) In RCC organ cultures, TNFR2 ligation by a TNFR2-selective mutein leads to activation of Etk and apparent transactivation of VEGFR2, promotes entry into the cell cycle and activates $\mathrm{NF}-\kappa \mathrm{B}$ in mTECs, whereas TNFR1 ligation by a TNFR1selective mutein causes an increase in TNFR1 and activation of $A S K 1$, promotes apoptosis, and activates NF- $\kappa$ B in mTECs. wtTNF activates both sets of responses. iv)
A
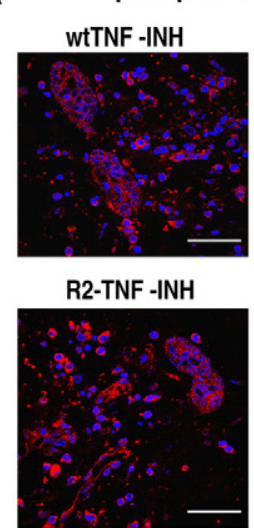

VEGF -INH

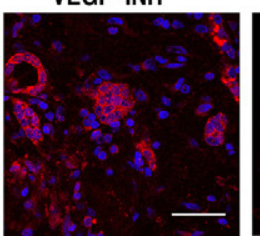

B

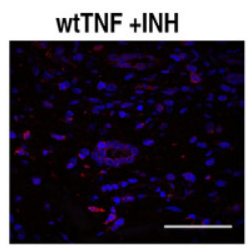

R2-TNF + INH

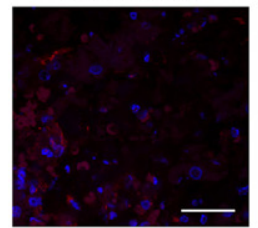

VEGF + INH

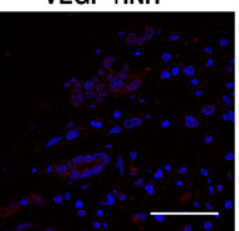

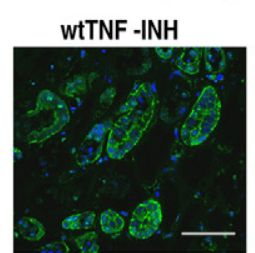

R2-TNF -INH

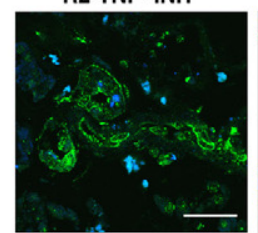

VEGF -INH

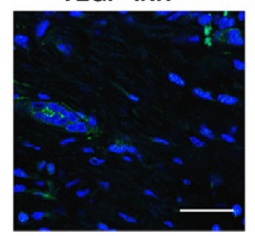

phospho-Etk ${ }^{\text {Tyr40 }}$

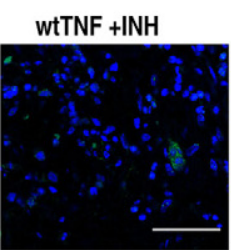

R2-TNF + INH

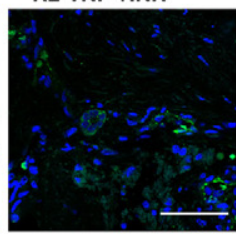

VEGF +INH

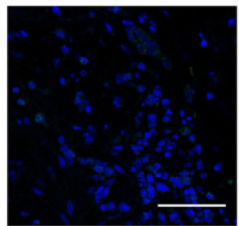

C
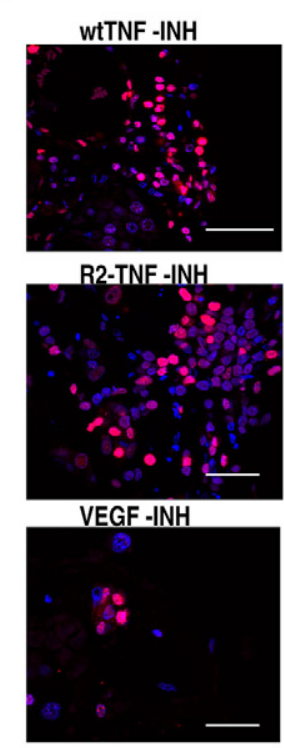

H3phospho-S10

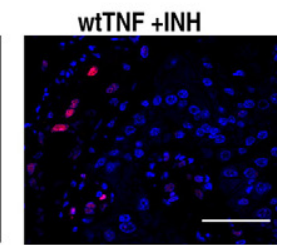

R2-TNF + INH

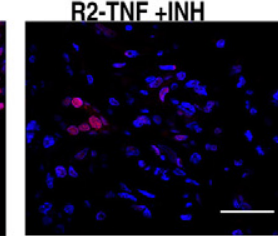

VEGF +INH

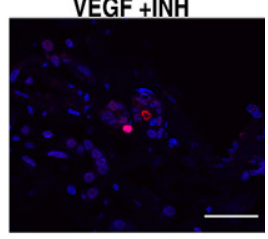

Figure 8. Confocal images showing expression of phospho-VEGFR2 ${ }^{\text {pY1054-1059 }}$, phospho-Etk ${ }^{\text {pTyr40 }}$, and H3 ${ }^{\text {phospho-S10 }}$ in ccRCC organ cultures. A: wtTNF, R2-TNF,

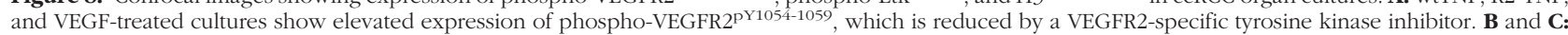
In contrast, wtTNF and R2-TNF, but not VEGF, induce activation of Etk and H3 $3^{\text {phospho-S10 }}$ also reduced by a VEGFR2-specific tyrosine kinase inhibitor. -INH, without inhibitor; +INH, with inhibitor. Images are representative of six different organ cultures with similar results. Scale bars $=20-200 \mu \mathrm{m}$. 
The kinetics of caspase activation and cell cycle entry are slower than those of ASK1, Etk, and VEGFR2 phosphorylation, consistent with a role for these kinases in these cellular events. v) TNFR2 effects on cell cycle entry are mediated through its interactions with Etk and VEGFR2 as a VEGFR2-selective tyrosine kinase inhibitor diminished the response to TNFR2 as well as to VEGF. vi) The signals induced by TNFR2 and VEGFR2 are distinct because only the former leads to activation of Etk. Taken together, these observations suggest that TNF signaling throughTNFR2 plays an important role in regulation and progression of RCC tumors.

The observed TNFR2-dependent up-regulation of TNFR2 expression and TNFR2-mediated activation of phospho-Etk ${ }^{\text {TTyr40 }}$ and -VEGFR2 $2^{\text {PY1054-59 }}$ in mTECs in ccRCC organ culture is important, because it implies that TNFR2 constitutes an initial signal for Etk-VEGFR2 activation. This hypothesis is supported by previous in vitro observations in endothelial cells, ${ }^{4}$ and our data indicate that a similar mechanism may be operative in MTECs in situ in RCC, where TNF and VEGF induce phosphorylation of VEGFR2 at Tyr(P)-1054-1059. However, in contrast to wtTNF and a TNFR2-selective mutein, VEGF did not induce activation of Etk or induction of $\mathrm{H} 3^{\text {phospho-S10 }}$ and $\mathrm{Ki}-67$ in our ccRCC organ cultures, suggesting that signals induced by ligation of TNFR2 and VEGFR2 are distinct and that Etk is important for the proliferative response in mTECs. Interestingly, Etk has been shown to control the proliferation of mammary epithelial cancer cells. ${ }^{29}$ It is worth noting that our studies are based on experiments in organ culture where cell viability is of limited duration and we cannot readily measure cell division, a relatively lengthy process. However, the change in nuclei showing markers of cell cycle progression between treated and untreated cultures is a more dynamic marker than simply counting nuclei that show evidence of cell cycle activation. Ki-67 as a marker of cell cycle entry has been shown to be an important prognostic indicator for RCC. ${ }^{30}$

Blockade of the VEGF pathway offers a new therapeutic approach to the treatment of RCC, ${ }^{31}$ and we have shown that both TNF- and VEGF-induced phosphorylation of VEGFR2 in mTECs is significantly diminished by a VEGFR2-selective tyrosine kinase inhibitor. Although it is possible that SU1498 blocks TNFR2 responses by effects on an as yet unidentified tyrosine kinase, its effects on TNF-induced phosphorylation of VEGFR2, Etk phosphorylation, and TNF-induced cell cycle entry support the role of TNFR2-mediated Etk-VEGFR2 activation in mTEC proliferation.

TNFR2 may also promote RCC progression through initiation of other TNF signaling pathways. TNF can initiate apoptosis through formation of TNF receptor-associated factor-2 (TRAF2)-dependent formation of a death-inducing signaling complex or TRAF2-dependent activation of ASK1. These responses are thought to be predominantly activated by TNFR1 and can explain the increased cell death seen in mTECs after TNFR1 ligation. Apoptosis signaling pathways are held in check by a number of antiapoptotic proteins, including cFLICE (procaspase-8) inhibitory protein (c-FLIP), cellular inhibitors of apoptosis (c-IAPs), A20, and mitogen- activated protein kinase-specific phosphatases. Transcription of these proteins is induced by NF- $\kappa \mathrm{B}$, which is effectively activated by a TNFR2-selective mutein in mTEC. This demonstration of TNFR2-induced NF- $\kappa \mathrm{B}$ activation in mTECs is a somewhat unexpected finding because, unlike TNFR1, TNFR2 is generally a poor activator of this signaling pathway despite its high TRAF2 binding capability. ${ }^{32}$ Interestingly, studies have shown that exogenous TNF-induced activation of caspase-3 is suppressed in RCC lacking functional $\mathrm{pVHL}$, which display increased nuclear translocation of NK- $\kappa \mathrm{B}$ and increased expression of antiapoptotic genes including C-FLIP, survivin, C-IAP-1, and C-IAP-2. ${ }^{33}$

A final point of discussion is that the present report makes use of a human tumor organ culture system to study the responses of human cancer cells in their natural tissue environment. We have noted that cultured cells often fail to replicate the behaviors of cell populations in situ. For human tumors, which cannot be readily manipulated in living patients, the organ culture approach, analyzed through a variety of morphological assays, allows both cellular responses and pharmacological interventions to be tested. This may be an important tool for establishing the relevance of specific pathways that have been elucidated from cell line studies.

In summary, our study demonstrates that TNF exerts distinct effects on mTECs through each of its two receptors and that these receptors are differentially expressed as a consequence of malignant transformation. In particular, TNF signaling through TNFR2 may act as an autocrine growth factor in ccRCC. Thus, strategies to reduce TNFR2 expression or to selectively block signaling through TNFR2 may be more effective in halting the progression of this tumor than global blockade of TNF. In addition, VEGFR kinase inhibitors may be more effective than VEGF neutralization because TNF-TNFR2 signaling may provide an alternative and possibly more effective VEGF-independent means to activate this mitogenic receptor.

\section{Acknowledgments}

We thank Dr. Sathia Thiru (consultant renal pathologist at Addenbrooke's Hospital, Cambridge, UK) for her help in ccRCC classification and identification of tumor cells. We also thank the Tissue Bank (Department of Histopathology, Addenbrooke's Hospital, Cambridge, UK) for proving us with the tissue.

\section{References}

1. Rini BI: Vascular endothelial growth factor-targeted therapy in renal cell carcinoma: current status and future directions. Clin Cancer Res 2007, 13:1098-1106

2. Galbán S, Fan J, Martindale JL, Cheadle C, Hoffman B, Woods MP Temeles G, Brieger J, Decker J, Gorospe M: von Hippel-Lindau protein-mediated repression of tumor necrosis factor alpha translation revealed through use of cDNA arrays. Mol Cell Biol 2003, 23:2316-2328

3. Maisey N: Antitumor necrosis factor (TNF- $\alpha$ ) antibodies in the treatment of renal cell cancer. Cancer Invest 2007, 25:589-593

4. Zhang R, Xu Y, Ekman N, Wu Z, Wu J, Alitalo K, Min W: Etk/Bmx 
transactivates vascular endothelial growth factor 2 and recruits phosphatidylinositol 3-kinase to mediate the tumor necrosis factor-induced angiogenic pathway. J Biol Chem 2003, 278:51267-51276

5. Al-Lamki RS, Wang J, Skepper JN, Thiru S, Pober JS, Bradley JR: Expression of tumor necrosis factor receptors in normal kidney and rejecting renal transplants. Lab Invest 2001, 81:1503-1515

6. Cohen HT, McGovern FJ: Renal-cell carcinoma. N Engl J Med 2005, 353:2477-2490

7. Kovacs G, Akhtar M, Beckwith BJ, Bugert P, Cooper CS, Delahunt B, Eble JN, Fleming S, Ljungberg B, Medeiros LJ, Moch H, Reuter VE, Ritz E, Roos G, Schmidt D, Srigley JR, Storkel S, van den BE, Zbar B: The Heidelberg classification of renal cell tumours. J Pathol 1997, 183:131-133

8. Fuhrman SA, Lasky LC, Limas C: Prognostic significance of morphologic parameters in renal cell carcinoma. Am J Surg Pathol 1982, 6:655-663

9. Guinan P, Sobin LH, Algaba F, Badellino F, Kameyama S, MacLennan G, Novick A: TNM staging of renal cell carcinoma: Workgroup No. 3. Union International Contre le Cancer (UICC) and the American Joint Committee on Cancer (AJCC), Cancer 1997, 80:992-993

10. Al-Lamki RS, Wang J, Tolkovsky AM, Bradley JA, Griffin JL, Thiru S, Wang EC, Bolton E, Min W, Moore P, Pober JS, Bradley JR: TL1A both promotes and protects from renal inflammation and injury. J Am Soc Nephrol 2008, 19:953-960

11. Al-Lamki RS, Wang J, Vandenabeele P, Bradley JA, Thiru S, Luo D, Min W, Pober JS, Bradley JR: TNFR1- and TNFR2-mediated signaling pathways in human kidney are cell type-specific and differentially contribute to renal injury. FASEB J 2005, 19:1637-1645

12. Van Ostade X, Vandenabeele P, Tavernier J, Fiers W: Human tumor necrosis factor mutants with preferential binding to and activity on either the R55 or R75 receptor. Eur J Biochem 1994, 220:771-779

13. Cameron RI, Ashe P, O'Rourke DM, Foster H, McCluggage WG: A panel of immunohistochemical stains assists in the distinction between ovarian and renal clear cell carcinoma. Int J Gynecol Pathol 2003, 22:272-276

14. McGregor DK, Khurana KK, Cao C, Tsao CC, Ayala G, Krishnan B, Ro JY, Lechago J, Truong LD: Diagnosing primary and metastatic renal cell carcinoma: the use of the monoclonal antibody 'Renal Cell Carcinoma Marker.' Am J Surg Pathol 2001, 25:1485-1492

15. Ortiz-Rey JA, Gomez de MC, Pelaez BE, Fernandez CA, Barbosa Barreiro MJ, Anton BI: Expression of CD10 and renal cell carcinoma marker in clear cell renal cell carcinoma: analysis on tissue arrays. Actas Urol Esp 2006, 30:281-286

16. Voraberger G, Schafer R, Stratowa C: Cloning of the human gene for intercellular adhesion molecule 1 and analysis of its $5^{\prime}$-regulatory region. Induction by cytokines and phorbol ester. J Immunol 1991, 147:2777-2786

17. Shindler KS, Roth KA: Double immunofluorescent staining using two unconjugated primary antisera raised in the same species. J Histochem Cytochem 1996, 44:1331-1335

18. Jones SJ, Ledgerwood EC, Prins JB, Galbraith J, Johnson DR, Pober JS, Bradley JR: TNF recruits TRADD to the plasma membrane but not the trans-Golgi network, the principal subcellular location of TNF-R1. J Immunol 1999, 162:1042-1048

19. Avery AK, Beckstead J, Renshaw AA, Corless CL: Use of antibodies to RCC and CD10 in the differential diagnosis of renal neoplasms. Am J Surg Pathol 2000, 24:203-210
20. Pan CC, Chen PC, Ho DM: The diagnostic utility of MOC31, BerEP4 RCC marker and CD10 in the classification of renal cell carcinoma and renal oncocytoma: an immunohistochemical analysis of 328 cases. Histopathology 2004, 45:452-459

21. Lovisolo JA, Casati B, Clerici L, Marafante E, Bono AV, Celato N, Salvadore M: Gene expression profiling of renal cell carcinoma: a DNA macroarray analysis. BJU Int 2006, 98:205-216

22. Geramizadeh B, Ravanshad M, Rahsaz M: Useful markers for differential diagnosis of oncocytoma, chromophobe renal cell carcinoma and conventional renal cell carcinoma. Indian J Pathol Microbiol 2008, 51:167-171

23. Zhou M, Roma A, Magi-Galluzzi C: The usefulness of immunohistochemical markers in the differential diagnosis of renal neoplasms. Clin Lab Med 2005, 25:247-257

24. Chuang MJ, Sun KH, Tang SJ, Deng MW, Wu YH, Sung JS, Cha TL, Sun GH: Tumor-derived tumor necrosis factor- $\alpha$ promotes progression and epithelial-mesenchymal transition in renal cell carcinoma cells. Cancer Sci 2008, 99:905-913

25. Falkensammer C, Johrer K, Gander H, Ramoner R, Putz T, Rahm A, Greil R, Bartsch G, Thurnher M: IL-4 inhibits the TNF- $\alpha$ induced proliferation of renal cell carcinoma (RCC) and cooperates with TNF- $\alpha$ to induce apoptotic and cytokine responses by RCC: implications for antitumor immune responses. Cancer Immunol Immunother 2006, 55:1228-1237

26. Harrison ML, Obermueller E, Maisey NR, Hoare S, Edmonds K, Li NF, Chao D, Hall K, Lee C, Timotheadou E, Charles K, Ahern R, King DM, Eisen T, Corringham R, DeWitte M, Balkwill F, Gore M: Tumor necrosis factor $\alpha$ as a new target for renal cell carcinoma: two sequential phase II trials of infliximab at standard and high dose. J Clin Oncol 2007, 25:4542-4549

27. MacLennan GT, Farrow GM, Bostwick DG: Low-grade collecting duct carcinoma of the kidney: report of 13 cases of low-grade mucinous tubulocystic renal carcinoma of possible collecting duct origin. Urology 1997, 50:679-684

28. Elsässer-Beile U, Kolble N, Grussenmeyer T, Wetterauer U, SchultzeSeemann W: Correlation of clinical and immunological parameters of metastatic renal cell carcinoma patients undergoing therapy with interleukin 2, interferon- $\alpha$ and retinoic acid. Anticancer Res 1998, 18:1883-1890

29. Bagheri-Yarmand R, Mandal M, Taludker AH, Wang RA, Vadlamudi RK, Kung HJ, Kumar R: Etk/Bmx tyrosine kinase activates Pak1 and regulates tumorigenicity of breast cancer cells. J Biol Chem 2001, 276:29403-29409

30. Aaltomaa S, Lipponen P, la-Opas M, Eskelinen M, Syrjanen K: Prognostic value of Ki-67 expression in renal cell carcinomas. Eur Urol 1997, 31:350-355

31. Escudier B, Cosaert J, Pisa P: Bevacizumab: direct anti-VEGF therapy in renal cell carcinoma. Expert Rev Anticancer Ther 2008, 8:1545-1557

32. Rothe M, Wong SC, Henzel WJ, Goeddel DV: A novel family of putative signal transducers associated with the cytoplasmic domain of the $75 \mathrm{kDa}$ tumor necrosis factor receptor. Cell 1994, 78:681-692

33. Qi H, Ohh M: The von Hippel-Lindau tumor suppressor protein sensitizes renal cell carcinoma cells to tumor necrosis factor-induced cytotoxicity by suppressing the nuclear factor- $\kappa \mathrm{B}$-dependent antiapoptotic pathway. Cancer Res 2003, 63:7076-7080 